\title{
A pest management model with state feedback control
}

\author{
Qiong Liu ${ }^{1 *}$, Lizhuang Huang ${ }^{2}$ and Lansun Chen ${ }^{3}$
}

"Correspondence: lq082016@163.com

${ }^{1}$ College of Mathematics and Computer Science, Qinzhou University, Qinzhou, 535000, People's Republic of China

Full list of author information is available at the end of the article

\begin{abstract}
In this paper, research of a class of state feedback control model which is mainly used in crop pests management, with Bendixson-Dulac discriminance, proves that this model has an unique and globally stable positive equilibrium under the weak time-delay kernel function. Also, we adopt the subsequent function method in the ordinary differential equation of the geometric theory to prove that a sufficient condition holds for the existence of an order one period solution in the system. At the same time, it also proves that the periodic solution is asymptotically stable.
\end{abstract}

Keywords: pest management; successor function; periodic solution of order one; Bendixson-Dulac discriminance

\section{Introduction}

Crop is an essential part of the human food resources for sustainable development. Therefore, crop yields directly affect social and economic development as well as social stability. In recent years, in order to improve the yield of crops and meet the needs of human survival, people used a lot of pesticide [1-4] to keep pest down to improve the yield of crops, but the excessive use of pesticides will make the pests drug-resistant, pollute the environment, and also do some harm to people and animals. So how to reduce the effect caused by pests but doing no harm to the living environment of human beings and animals is an issue which has attracted extensive concern of ecologists and biomathematics researchers. In [5-10], the authors established a specific pest management model based on the actual problem and analyzed pest management issues by the corresponding mathematical theory. We will explore the feasibility of the crop pest management from the mathematical model of state feedback control in this paper.

In recent years, the research on the pest management has made many achievements. It can be classified into two categories: one is using farm chemicals, but this method will strengthen the drug resistance of the pests and it is harmful to the environment. The other is adopting a biological treatment [11-13] such as taking advantage of the characteristics of inter-restriction between the beneficial organisms and pests, it can effectively maintain a long-term balance with the effects on the food chain. The latter treatment is a more extensive pest treatment at present. It is a better measure to control pest by cultivating natural enemies of the pests to destroy pests, but the delivery of natural enemies and use of pesticides are not regular, depending on the amount of pests. Therefore, we must give full consideration to the natural inhibition in the agricultural ecological system. Meanwhile,

(c) Liu et al. 2016. This article is distributed under the terms of the Creative Commons Attribution 4.0 International License (http://creativecommons.org/licenses/by/4.0/), which permits unrestricted use, distribution, and reproduction in any medium, provided you give appropriate credit to the original author(s) and the source, provide a link to the Creative Commons license, and indicate if changes were made. 
delivery of a natural enemy and use of pesticides are transient phenomena, which we call a pulse phenomenon. In biological control, we do not need to reduce the amount of pests to zero, but we usually control the indicator of the state of an illness under a certain indicator, and this cannot only boost the yields of crops, but also it has no negative influences on the environment. We call the indicator of the state of an illness the economic index (EI), also known as the control index.

The infinite delay logistic model of classic single species is expressed as

$$
\frac{d x(t)}{d t}=x(t)\left[a-b x(t)-c \int_{-\infty}^{t} k(t-s) x(s) d s\right] .
$$

Here, $x(t)$ is the population density at $t, a, b, c$ are positive constants, $a$ expresses the innate capacity of increase, $b, c$ express the density restriction coefficient, the integral kernel $k$ is continuous and meets $\int_{0}^{\infty} k(s) d s=1$ and $\int_{0}^{\infty} s k(s) d s<+\infty$. In system (1), when $b \neq 0$, one has a so-called non-pure time delay, and when $b=0$, it is called a pure time delay. Corresponding to system (1), Fengde Chen [14] also put forward the model of an infinite delay logarithmic population:

$$
\frac{d x(t)}{d t}=x(t)[a-b \ln x(t)-c k(t-s) \ln x(s) d s] .
$$

It has biological significance for the above system (1); and meets $\int_{0}^{\infty} k(s) d s=1$ and $\int_{0}^{\infty} s k(s) d s<+\infty$

In practical pest management, we do not periodically use the pesticide before we determine the pesticide. So, on the basis of system (2), this paper studies the impulsive state feedback control with continuous weak delay logarithm population,

$$
\begin{cases}\frac{d x(t)}{d t}=x(t)\left[a-b \ln x(t)-c \int_{-\infty}^{t} k(t-s) \ln x(s) d s\right], & x<h, \\ \Delta x=-\beta x, & x=h .\end{cases}
$$

Under the condition of a weak integral kernel, the given specific integral kernel $k(t-s)=$ $e^{-d(t-s)}$, we make a transformation, assuming $u(t)=\ln x(t), v(t)=\int_{-\infty}^{t} e^{-d(t-s)} \ln x(s) d s$; then

$$
\begin{aligned}
& u^{\prime}(t)=\frac{1}{x(t)} x^{\prime}(t)=a-b \ln x(t)-c \int_{-\infty}^{t} k(t-s) \ln x(s) d s=a-b u-c v, \\
& v^{\prime}(t)=\left(e^{-d t} \int_{-\infty}^{t} e^{d s} \ln x(s) d s\right)^{\prime}=-d v+u, \\
& x\left(t^{+}\right)-x(t)=\Delta x=-\beta x, \quad x\left(t^{+}\right)-x(t)=\Delta x=-\beta x, \\
& x\left(t^{+}\right)-x(t)=\Delta x=-\beta x, \quad \Delta u=u\left(t^{+}\right)-u(t)=-\beta \ln h .
\end{aligned}
$$

System (3) turns into the following system:

$$
\left\{\begin{array}{ll}
\frac{d u}{d t}=a-b u-c v, \\
\frac{d v}{d t}=u-d v, \\
\Delta u=-\beta u,
\end{array}\right\} \begin{aligned}
& u<\ln h \\
& u=\ln h
\end{aligned}
$$

Among them, $u$ refers to crop pest density, $v$ refers to the hysteresis effect of the pest, $\ln h$ is the economic index, $a$ means the intrinsic rate of increase, $b$ and $c$ are density restriction 
factors, $d$ is positive constant, which refers to the parameters of the normal distribution, $\int_{0}^{\infty} e^{s} d s=1,0<\beta<1$ is the ratio of spraying pesticide pests.

\section{Preparation}

First of all the related definition is given [15].

Consider the state impulsive differential equations

$$
\left\{\begin{array}{lll}
\frac{d x}{d t}=f(x, y), & \frac{d y}{d t}=g(x, y), & (x, y) \in M(x, y), \\
\Delta x=\alpha(x, y), & \Delta y=\beta(x, y), & (x, y) \notin M(x, y) .
\end{array}\right.
$$

Here, $M(x, y)$ and $N(x, y)$ are straight lines or curves on the plane $R^{2}(x, y), M(x, y)$ is the pulse set, $N(x, y)$ is the phase set, $\varphi(M)=N$, system (5) is called a constituted power system. It is a straight line or curve in $R_{2}^{+}=\left\{(x, y) \in R^{2} \mid x \geq 0, y \geq 0\right\}$.

Definition 2.1 Assume $M$ is the pulse set, $N$ is the phase set, and $M$ and $N$ are both straight lines, as shown in Figure 1. Assume the coordinate of the point of intersection $Q$ of $N$ and $x$ is $O$, and the distance between $E$ and $Q$ on the line $N$ is denoted $c$. The path line through the point $E$ intersects with the pulse set at point $G$, the phase point of the point $G$ in the phase set $N$ is $F$, and the coordinate is $d$. We define point $F$ to be the subsequent point of the point $E$ and the successor function of the point $E$ is $G(E)=d-c$.

For convenience in our discussion, the following definitions are used in this article in terms of the subsequent function.

Definition 2.2 The point of intersection between the path line $L$ and the phase set $u=$ $(1-\beta) \ln h$ is $N$, the point of intersection between the path line $L$ and the pulse set $u=\ln h$ is impulsing and its $v$ coordinate difference to the phase point $N_{1}$ on the phase set $u=$ $(1-\beta) \ln h, G(N)=N_{1 v}-N_{v}$.

Lemma 2.1 [16] The subsequent function $G(E)$ is continuous.

Lemma 2.2 Assume in the continuous dynamical system $(X, \Pi)$, there are two points, $x_{1}$, $x_{2}$ in pulse phase set, to make the successor functions, $G\left(x_{1}\right)>0, G\left(x_{2}\right)<0$, then there must be a point $E$ between $x_{1}$ and $x_{2}$ to make $G(E)=0$. Thus, there must be a periodic solution of order one through $E$ between $x_{1}$ and $x_{2}$.

Figure 1 The schematic diagram with $F$ as subsequent point of $G$.

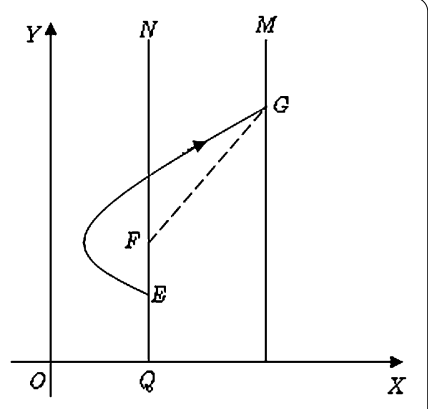


Lemma 2.3 (Similar Poincaré norm) Assume the periodic solutions of $T$ - in the following system are $x=\varphi(t), y=\phi(t)$ :

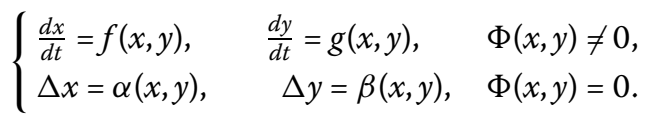

The path asymptotically stable. Assume the multiplier $u_{2}$ meets $\left|u_{2}\right|<1$. Here,

$$
\begin{gathered}
u_{2}=\prod_{k=1}^{q} \Delta_{k} \exp \left[\int_{0}^{T}\left(\frac{\partial f}{\partial x}(\varphi(t), \phi(t))+\frac{\partial g}{\partial y}(\varphi(t), \phi(t))\right) d t\right], \\
\Delta_{k}=\frac{f_{+}\left(\frac{\partial \beta}{\partial y} \cdot \frac{\partial \Phi}{\partial x}-\frac{\partial \beta}{\partial x} \cdot \frac{\partial \Phi}{\partial y}+\frac{\partial \Phi}{\partial x}\right)+g_{+}\left(\frac{\partial \alpha}{\partial x} \cdot \frac{\partial \Phi}{\partial y}-\frac{\partial \alpha}{\partial y} \cdot \frac{\partial \Phi}{\partial x}+\frac{\partial \Phi}{\partial y}\right)}{f\left(\frac{\partial \Phi}{\partial x}\right)+g\left(\frac{\partial \Phi}{\partial y}\right)}
\end{gathered}
$$

and $f, g, \frac{\partial \alpha}{\partial x}, \frac{\partial \alpha}{\partial y}, \frac{\partial \beta}{\partial x}, \frac{\partial \beta}{\partial y}, \frac{\partial \Phi}{\partial x}, \frac{\partial \Phi}{\partial y}$ the value at the point $\left(\varphi\left(\tau_{k}\right), \phi\left(\tau_{k}\right)\right)$,

$$
f_{+}=f\left(\varphi\left(\tau_{k}^{+}\right), \phi\left(\tau_{k}^{+}\right)\right), \quad g_{+}=g\left(\varphi\left(\tau_{k}^{+}\right), \phi\left(\tau_{k}^{+}\right)\right) .
$$

Lemma 2.4 (Bendixson-Dulac discriminance [17]) Consider the system

$$
\left\{\begin{array}{l}
\frac{d x}{d t}=P(x, y), \\
\frac{d y}{d t}=Q(x, y) .
\end{array}\right.
$$

If in a connected area $D, P(x, y)$ and $Q(x, y)$ have continuous partial derivatives, $\frac{\partial P}{\partial x}+\frac{\partial Q}{\partial y}$ maintains a constant value and it is zero all the time in any subdomain, then system (6) has no closed trajectory in D.

\section{Existence and stability of the periodic solution of order one for the impulsive differential equation of plant diseases and insect pests}

In system (4), if $\beta=0$, then we will obtain a mathematical model for control of the class-A crops diseases and insect pests without pulse,

$$
\left\{\begin{array}{l}
\frac{d u}{d t}=a-b u-c v \\
\frac{d v}{d t}=u-d v
\end{array}\right.
$$

If $\beta>0$, then system (4) obeys the following.

Lemma 3.1 The positive equilibrium point of system $(4) E\left(u^{*}, v^{*}\right)=E\left(\frac{a d}{c+b d}, \frac{a}{c+b d}\right)$ has global stability.

Proof Assume

$$
\left\{\begin{array}{l}
\frac{d u}{d t}=a-b u-c v \equiv P(u, v), \\
\frac{d v}{d t}=u-d v \equiv Q(u, v) .
\end{array}\right.
$$

As a result the system's variational matrix is

$$
\left(\begin{array}{ll}
\frac{\partial P}{\partial u} & \frac{\partial P}{\partial v} \\
\frac{\partial Q}{\partial u} & \frac{\partial Q}{\partial v}
\end{array}\right)=\left(\begin{array}{cc}
-b & -c \\
1 & -d
\end{array}\right) .
$$


So, the characteristic equation in the $E\left(u^{*}, v^{*}\right)$ is

$$
\left|\begin{array}{cc}
\lambda+b & c \\
-1 & \lambda+d
\end{array}\right|=(\lambda+b)(\lambda+d)+c=0
$$

Namely, $\lambda^{2}+\lambda(b+d)+b d+c=0$.

We obtain $\lambda_{1,2}=\frac{-(b+d) \pm \sqrt{(b+d)^{2}-4(b d+c)}}{2}$, in which $b, d, c$ are all constant.

Assume $\Delta=T^{2}-4 D=(b+d)^{2}-4(b d+c), T=-(b+d), D=b d+c$.

(1) When $\Delta=0, T<0, D>0$, the two characteristic roots $\lambda_{1}=\lambda_{2}$ is a multiple negative real root, at this point, the solution curve tends to equilibrium, called the degenerate node.

(2) When $\Delta>0, T<0, D>0$, the two characteristic roots $\lambda_{1} \neq \lambda_{2}$ is a pair of negative real roots, at this point, the solution curve tends to equilibrium, called the stable node.

(3) When $\Delta<0, T<0, D>0$, the two characteristic roots $\lambda_{1}, \lambda_{2}$ have real parts and they are negative conjugate complex roots, at this point, the solution curve tends to equilibrium, and the equilibrium is called a focal point.

From the above analysis, we can see the characteristic roots shall be two conjugate complex roots of which two are negative or a pair with a negative real part and this shows that the positive equilibrium point $E\left(u^{*}, v^{*}\right)^{T}$ is locally stable. It can also be noticed that on the $R^{2}$ plane, constantly

$$
\frac{\partial P}{\partial u}+\frac{\partial Q}{\partial v}=-(b+d)<0 .
$$

From Lemma 2.4 we know that on the whole plane the system has no limit cycle, and because there is only a balance in the system, so all the path lines take $E\left(u^{*}, v^{*}\right)^{T}$ as the limit set, the system is stable at the balance point $E\left(u^{*}, v^{*}\right)^{T}$ and on the plane $R^{2}$. It is hereby proved.

When the positive constants $b, c, d$ meet $(b+d)^{2}<4(b d+c)$, the positive equilibrium is a stability focal point, namely when the real part of the two characteristic roots $\lambda_{1}, \lambda_{2}$ are negative conjugate complex roots, of which $v_{1}=\frac{a-b \ln h}{c}$ is the intersection point $D\left(\ln h, v_{1}\right)$ between the pulse set $u=\ln h$ and the line $\frac{d u}{d t}=0$, and when $h \geq 1$, we have the following theorem.

\section{Theorem 3.1}

(1) If the pulse set $0<\ln h \leq \frac{a d}{c+b d}$, then system (4) has a periodic solution of the order one.

(2) If the pulse set $\ln h>\frac{a d}{c+b d}>0$, there are the following four conditions:

(i) When $c_{1 v}<v_{1}, c_{2 v}<v_{1}$, system (4) has a periodic solution of order one.

(ii) If the subsequent point $B_{1}$ overlaps $B$, the other path line $\Gamma_{1}$ has no intersection point with the pulse set, then system (4) has a periodic solution of order one.

(iii) If $c_{2 v}<v_{1}$, the system path line $\Gamma_{1}$ has no intersection point with the pulse set $\ln h$, then system (4) has no periodic solution of order one, but for any $t$, we have $v(t) \leq v_{1}+\frac{b \beta \ln h}{c}$.

(iv) If the system path lines $\Gamma_{1}, \Gamma_{2}$ have no intersection point with the pulse set $\ln h$, then system (4) has no periodic solution of order one, but, for any $t$, we have $v(t) \leq v_{1}+\frac{c \ln h \beta}{c}$. 


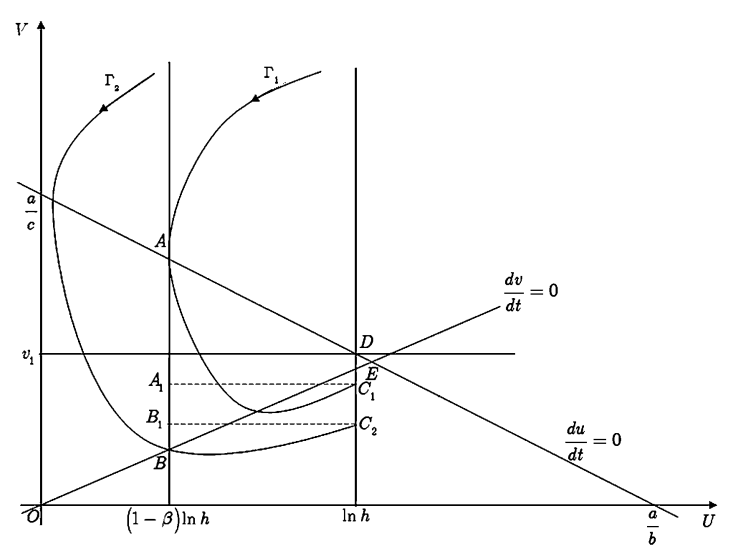

Figure 2 When $\ln h<\frac{a d}{c+b d}$, there is a periodic solution of order one in the diagram.

Proof (1) Assume the pulse set $\ln h \leq u^{*}=\frac{a d}{c+b d}$, namely $v_{1}>v^{*}=\frac{a}{c+b d}$, like Figure 2.

From the equation $\left\{\begin{array}{l}u=\ln h, \\ a-b u-c v=0,\end{array}\right.$, we obtain $v_{1}=\frac{a-b \ln h}{c}$.

From the equation $\left\{\begin{array}{l}u=(1-\beta) \ln h, \\ a-b u-c v=0,\end{array}\right.$, we obtain $v=\frac{a-b u}{c}=\frac{a-b(1-\beta) \ln h}{c}=v_{1}+\frac{b \beta \ln h}{c}$.

So, the system trajectory $\Gamma_{1}$ passing through the point $A\left((1-\beta) \ln h, v_{1}+\frac{b \beta \ln h}{c}\right)$ in the phase set $(1-\beta) \ln h$ intersects with the pulse set $\ln h$ at $C_{1}\left(\ln h, c_{1 v}\right)$, and this results in a pulse action in the phase set $u=(1-\beta) \ln h$, the phase point is $A_{1}\left((1-\beta) \ln h, c_{1 v}\right)$, obviously, the vertical coordinate of the point $A_{1}$ is less than that of the point $A$, we have

$$
G(A)=A_{1 v}-A_{v}=c_{1 v}-\left(v_{1}+\frac{c \ln h \beta}{c}\right)<0
$$

Assume $u=(1-\beta) \ln h$ intersects with $\frac{d v}{d t}=0$ at $B\left((1-\beta) \ln h, \frac{(1-\beta) \ln h}{d}\right)$, then select the system path line $\Gamma_{2}$ passing the point $B$ intersects with the pulse set $\ln h$ at $C_{2}\left(\ln h, c_{2 v}\right)$ and results in a pulse action in the phase set $u=(1-\beta) \ln h$ at $B_{1}\left((1-\beta) \ln h, c_{2 v}\right)$. And because the path line $\Gamma_{2}$ 's segmental arc is below the straight line $\frac{d v}{d t}=0$, so on the segmental arc $B C_{2}$ we have $\frac{d v}{d t}>0$, namely $v$ monotonically increases, with the nature of monotonic function we can obtain $c_{2 v}>\frac{(1-\beta) \ln h}{d}$, so there is a successor function $G(B)=B_{1 v}-B_{v}=$ $c_{2 v}-\frac{(1-\beta) \ln h}{d}>0$.

According to Lemma 2.2, in the phase set $u=(1-\beta) \ln h$, there must be $P$ which meets $B_{v}<P_{v}<A_{v}$, to make $G(P)=P_{v}-P_{1 v}=0$, namely there is a periodic solution of order one in system (4).

With the same method, we can prove that when $\ln h=\frac{a d}{c+b d}$, system (4) has a periodic solution of order 1 , like Figure 3 . Select the path line $\Gamma_{1}$ intersecting with the pulse set $(1-\beta) \ln h$ at $A\left((1-\beta) \ln h, \frac{b \beta \ln h}{c}\right)$, and intersecting with the pulse set at $C_{1}\left(\ln h, c_{1 v}\right)$, the phase point after the pulse is $A_{1}\left((1-\beta) \ln h, c_{1 v}\right)$, as the path line $\Gamma_{1}$ is below the $\frac{d u}{d t}=0$, the segmental arc above the $\frac{d v}{d t}=0$ has $\frac{d v}{d t}<0$, namely the $v$ monotonically decreases, therefore, the vertical coordinate of the point $A_{1}$ is less than that of the point $A$, we have $G(A)=A_{1 v}-A_{v}=c_{1 v}-\left(v_{1}+\frac{c \beta \ln h}{c}\right)<0$. In the same way, select the path line $\Gamma_{2}$ intersecting with the pulse set $(1-\beta) \ln h$ at the point $B\left((1-\beta) \ln h, \frac{(1-\beta) \ln h}{d}\right)$ and intersecting with the pulse set at $C_{2}\left(\ln h, c_{2 v}\right)$, the phase point after the pulse is $B_{1}\left((1-\beta) \ln h, c_{2 v}\right)$, and because the segmental arc $B C_{2}$ of the path line $\Gamma_{2}$ is below the straight line $\frac{d v}{d t}=0$, in $B C_{2}, \frac{d v}{d t}>0$, namely $v$ monotonically increases, with the nature of 


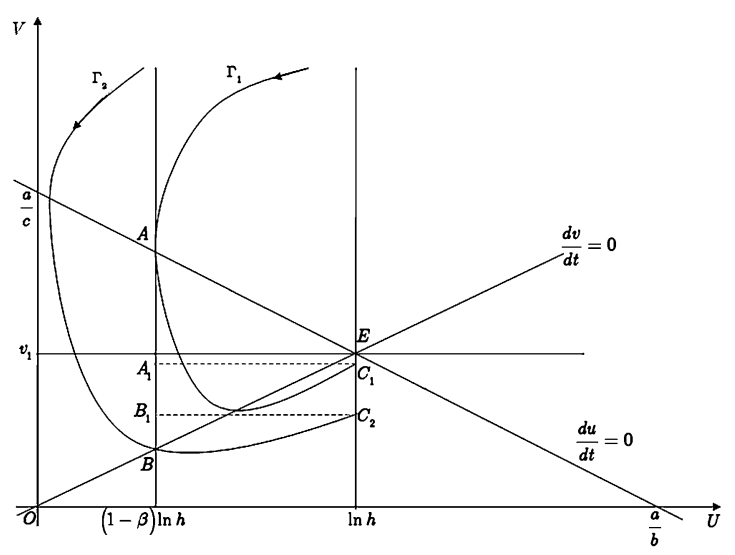

Figure 3 When $\ln h=\frac{a d}{c+b d}$, there is a periodic solution of order one in the diagram.

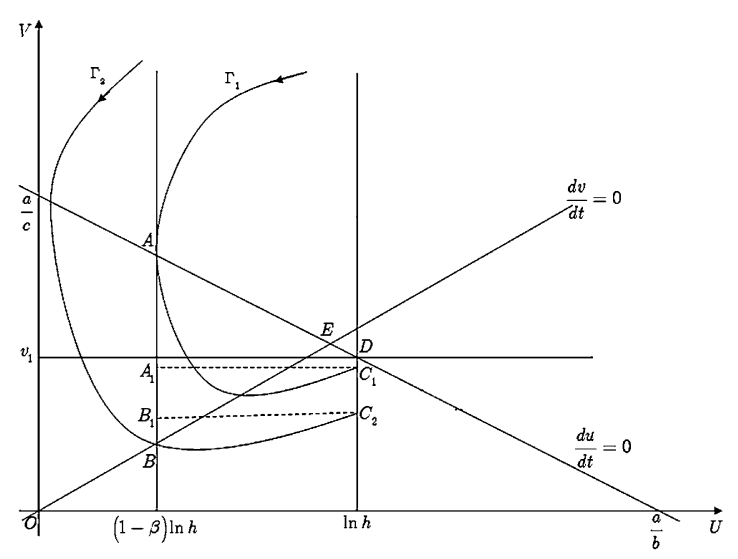

Figure 4 Meeting (i) a periodic solution of order one in the diagram.

the monotonic function, we can obtain $c_{2 v}>\frac{(1-\beta) \ln h}{d}$, so the successor function of $B$ obeys $G(B)=B_{1 v}-B_{v}=c_{2 v}-\frac{(1-\beta) \ln h}{d}>0$.

According to Lemma 2.2, in the phase set $u=(1-\beta) \ln h$, there must be $\exists P(P)$, this meets $B_{v}<P_{v}<A_{v}$, and it enables $G(P)=P_{v}-P_{1 v}=0$, namely there is a periodic solution of order one in system (4).

(2) If the pulse set obeys $\ln h>\frac{a d}{c+b d}$, under this condition, there are four situations as follows.

When $(1-\beta) \ln h<u^{*}=\frac{a d}{c+b d}<\ln h$, namely $v_{1}<v^{*}=\frac{a}{c+b d}<v_{1}+\frac{c \beta \ln h}{c}$.

(i) If $c_{1 v}<v_{1}, c_{2 v}<v_{1}$, then system (4) has a periodic solution of order one, as shown in Figure 4. Assume the intersection point between the pulse set $\ln h$ and the line $\frac{d u}{d t}=a-b u-c v=0$ is $D$, the coordinate of $D$ is solved by the following equation set:

$$
\left\{\begin{array}{l}
u=\ln h \\
a-b u-c v=0 .
\end{array}\right.
$$


We can obtain

$$
\left\{\begin{array}{l}
u=\ln h \\
v=\frac{a-b \ln h}{c} .
\end{array}\right.
$$

So for point $D\left(\ln h, \frac{a-b \ln h}{c}\right)$, we can select the path line $\Gamma_{1}$ passing the intersection point $A$ between the phase set $(1-\beta) \ln h$ and the line $\frac{d u}{d t}=0$, the coordinate of the point $A$ is $A\left((1-\beta) \ln h, v_{1}+\frac{b \beta \ln h}{c}\right)$, intersecting with the pulse set at $C_{1}$ and acts on the phase set with the pulse action, the phase point is $A_{1}$, the segmental arc of the path line $\Gamma_{1}$, $\frac{d v}{d t}<0$, is below the line $\frac{d u}{d t}=0$ and above $\frac{d v}{d t}=0$, so the $v(t)$ monotonically decreases. Therefore, according to the nature of the monotonic function, we can obtain a vertical coordinate of the point $A$ that is greater than that of the point $A_{1}$, namely $A_{v}>$ $A_{1 v}$, so the successor function of $A$ is $G(A)=A_{1 v}-A_{v}<0$. In the same way, select the path line $\Gamma_{2}$ passing the intersection point $B$ between the phase set $(1-\beta) \ln h$ and the line $\frac{d u}{d t}=0$, intersecting with the pulse set at $C_{2}$, the phase point of the corresponding phase set after the pulse is $B_{1}$, because the segmental arc $B C_{2}$ of the path line $\Gamma_{2}$ is below the line $\frac{d v}{d t}=0, \frac{d v}{d t}>0$, we can thus know the $v(t)$ monotonically increases, with the nature of the monotonic function, we can obtain vertical coordinate of the point $B$ is less than that of the point $B_{1}$, namely $B_{1 v}>B_{v}$, so the successor function of $B$ is $G(B)=B_{1 v}-B_{v}>0$.

According to Lemma 2.2, in the phase set $u=(1-\beta) \ln h, P$ must meet $B_{v}<P_{v}<A_{v}$, and this enables $G(P)=P_{v}-P_{1 v}=0$, namely there is a periodic solution of order one in system (4).

(ii) If the path line passes the $\Gamma_{2}$ and the point $B_{1}$ of the phase set, intersects with the point $B$ in the pulse set (the point $B$ coincides $D$ ), through the pulse action, the subsequent point of $B_{1}$ coincides $B_{1}$, that is, the $\Gamma_{2}$ parts monotonically increase and decrease on both sides of the line $\frac{d v}{d t}=0$ are equal, and with Lemma 2.2, we must have $G\left(B_{1}\right)=0$, at the same time, the other path line $\Gamma_{1}$ has no intersection point with the pulse set, then there is a periodic solution of order one in system (4). This is shown in Figure 5.

(iii) If $c_{2 v}<v_{1}$, the system's path line $\Gamma_{1}$ has no intersection points with the pulse set $\ln h$, then there is no periodic solution of order one in system (4), but for any $t$, we have $v(t) \leq$ $v_{1}+\frac{b \beta \ln h}{c}$, as shown in Figure 6. Due to considering the actual biological significance, the path line does not intersect with the pulse line, indicating there are not many pests, with

Figure 5 Meeting (ii) a periodic solution of order one.

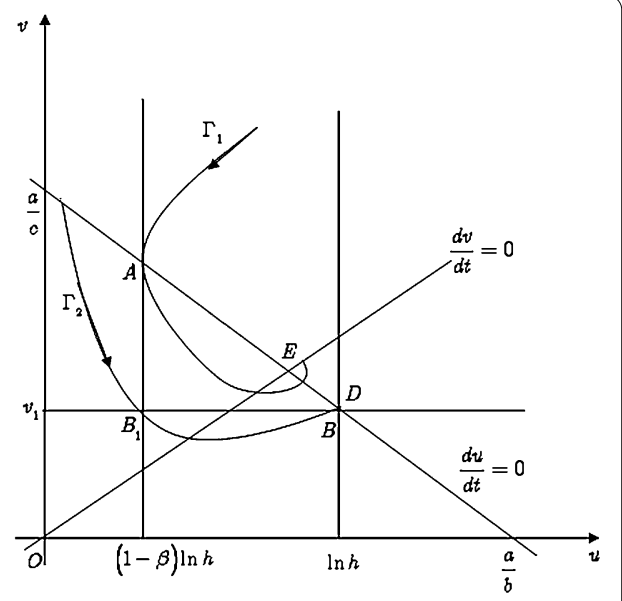


Figure 6 Meeting (iii) schematically.

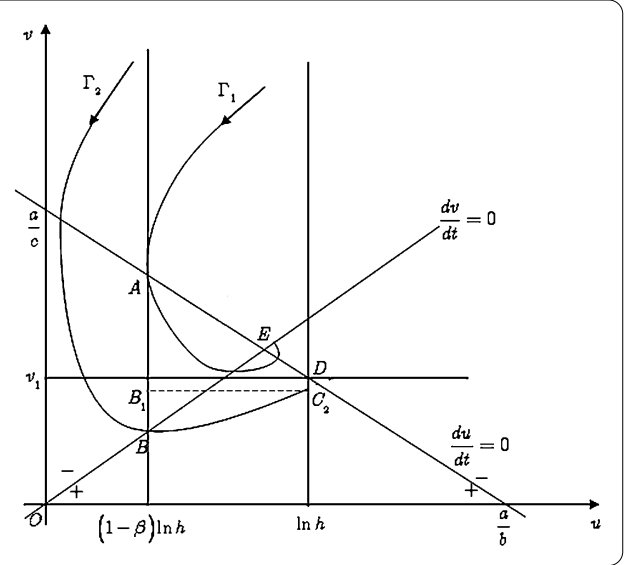

Figure 7 Meeting (iv) schematically.

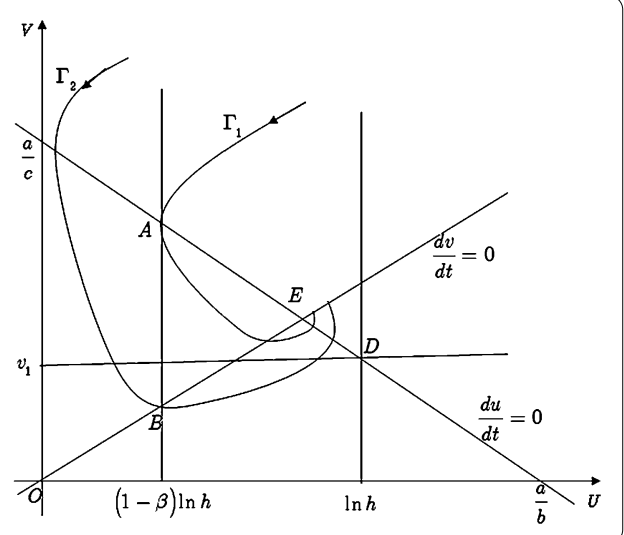

little harm to crops, so it cannot be controlled, that is to say, we can always find a path line cut in the phase set $A$, and the path line is fully near the pulse set, but it has no intersection with the pulse to constitute one biggest attraction domain, all of the path lines eventually arrive at the traction domain, with no intersection with pulse set, we do not need pulse control, pest populations in this area cannot meet the monitoring limit. Therefore, there is no periodic solution of order one. But any constituted attraction domain has $v(t) \leq$ $v_{1}+\frac{b \ln h \beta}{c}$ for any $t$.

(iv) If the system path lines $\Gamma_{1}, \Gamma_{2}$ have no intersection point with pulse set $\ln h$, then there is no periodic solution of order one in system (4), but for any $t$, we have $v(t) \leq v_{1}+$ $\frac{b \ln h \beta}{c}$, as shown in Figure 7. There is no intersection point between the path line and the pulse set, not meeting the definition of the periodic solution of order one, so there is no periodic solution of order one. Considering the actual biological significance, the analysis is the same as (iii). Theorem 3.1 is proved.

When $0<h<1$, namely the pulse set $\ln h<0<\frac{a d}{c+b d}$, then there is no periodic solution of order one in system (4). This case has no real biological meaning, so it is no longer considered.

Theorem 3.2 When $h \geq 1$ and $(b+d)^{2}<4(b d+c)$, the positive equilibrium is a stable focus and $a-c \phi_{0}<b(1-\beta) \ln h$, then the periodic path line I of order one in system (4) passing the point $\left(\ln h, \phi_{0}\right)$ is asymptotically stable. 
Proof By system (4) we can obtain

$$
\begin{aligned}
& \frac{\partial f}{\partial u}=-b, \quad \frac{\partial g}{\partial v}=-d, \quad \frac{\partial \alpha}{\partial u}=-\beta, \\
& \frac{\partial \beta}{\partial v}=0, \quad \Phi(u, v)=u-\ln h, \quad \frac{\partial \Phi}{\partial u}=1, \\
& \frac{\partial \Phi}{\partial \nu}=0, \quad(\varphi(T), \phi(T))=\left(\ln h, \phi_{0}\right), \\
& \left(\varphi\left(T^{+}\right), \phi\left(T^{+}\right)\right)=\left((1-\beta) \ln h, \phi_{0}\right), \\
& \Delta_{1}=\frac{f_{+}\left(\frac{\partial \beta}{\partial v} \cdot \frac{\partial \Phi}{\partial u}-\frac{\partial \beta}{\partial u} \cdot \frac{\partial \Phi}{\partial v}+\frac{\partial \Phi}{\partial u}\right)+g_{+}\left(\frac{\partial \alpha}{\partial u} \cdot \frac{\partial \Phi}{\partial v}-\frac{\partial \alpha}{\partial v} \cdot \frac{\partial \Phi}{\partial u}+\frac{\partial \Phi}{\partial v}\right)}{f\left(\frac{\partial \Phi}{\partial u}\right)+g\left(\frac{\partial \Phi}{\partial v}\right)} \\
& =\frac{f_{+}}{f}=\frac{f_{+}\left(\varphi\left(T^{+}\right), \phi\left(T^{+}\right)\right)}{f(\varphi(T), \phi(T))} \\
& =\frac{f_{+}\left((1-\beta) \ln h, \phi_{0}\right)}{f\left(\ln h, \phi_{0}\right)} \\
& =\frac{a-b(1-\beta) \ln h-c \phi_{0}}{a-b \ln h-c \phi_{0}} \text {, } \\
& \mu_{2}=\Delta_{1} \cdot \exp \left\{\int_{0}^{T}\left(\frac{\partial f}{\partial u}(\varphi, \phi)+\frac{\partial g}{\partial v}(\varphi, \phi)\right) d t\right\} \\
& =\Delta_{1} \cdot \exp \left\{\int_{0}^{T}[-(b+d)] d t\right\} \\
& =\Delta_{1} \cdot \exp \left\{\int_{(1-\beta) \ln h}^{\ln h}[-(b+d)] d t\right\} \\
& =\Delta_{1} \cdot \exp \{-\beta(b+d) \ln h\} \text {. }
\end{aligned}
$$

Assume $m=\beta(b+d) \ln h$, because $\beta, b, d$, are all positive constants greater than 0 , when $h \geq 1$, we have $\ln h \geq 0$, so $m \in[0,+\infty) . \forall m \in(0,+\infty)$, as $e^{-m}$ is a decreasing function, so $e^{-m} \leq 1$.

Take $\Delta_{1}$ into the following formula:

$$
\begin{aligned}
\mu_{2} & =\frac{a-b(1-\beta) \ln h-c \phi_{0}}{a-b \ln h-c \phi_{0}} e^{-m} \\
& \leq \frac{a-b(1-\beta) \ln h-c \phi_{0}}{a-b \ln h-c \phi_{0}} \\
& =1+\frac{b \beta \ln h}{a-b \ln h-c \phi_{0}} .
\end{aligned}
$$

When $h \geq 1, \mu_{2}=1+\frac{b \beta \ln h}{a-b \ln h-c \phi_{0}}=1-\frac{b \beta \ln h}{b \ln h+c \phi_{0}-a}$, we can see that when it meets $(b+d)^{2}<$ $4(b d+c)$, the positive equilibrium is a stable focus and $a-c \phi_{0}<b(1-\beta) \ln h$, namely when $a-c \phi_{0}<b(1-\beta) \ln h, 0<\mu_{2}<1$, so the path line $I$ of the order one cycle in system (4) is asymptotically stable.

Theorem 3.3 When $h \geq 1$ and $(b+d)^{2}<4(b d+c)$, the positive equilibrium is a stable focus and $a-c \phi_{0}<b(1-\beta) \ln h$, then the periodic path line I of the order one in system (4) is asymptotically stable. 


\section{Conclusion}

This paper focuses on an ecosystem model of class-A plant diseases and insect pests to analyze the stability of the equilibrium of the model; it discusses the sufficient condition for the existence of a periodic solution of order one of the system, and at the same time, it also proves the conditions under which the periodic solution is asymptotically stable. On the basis of the model, it carried on the qualitative and quantitate analysis, to draw some conclusions under certain conditions. That is to say, there is a periodic solution of order one in this paper and it is asymptotically stable, under this situation the number of pest is controlled to a certain extent, it will be finally controlled to a be a periodic solution of order one in which the once-occurring pulse finally controls the number of pest. It can be seen from the article that, according to the different crop growth cycle, the observation and record of the quantity of pests in production can help the crop-dusting to control pest populations, so as to protect crops.

\section{Competing interests}

The authors declare that they have no competing interests.

\section{Authors' contributions}

QL carried out the main part of this article, LC corrected the manuscript, LH brought forward some suggestions on this article. All authors have read and approved the final manuscript.

\section{Author details}

${ }^{1}$ College of Mathematics and Computer Science, Qinzhou University, Qinzhou, 535000, People's Republic of China. ${ }^{2}$ College of Mathematics and Information Science, Guangxi University, Nanning, 530004, People's Republic of China. ${ }^{3}$ Academy of Mathematics and System Sciences, Chinese Academy of Sciences, Beijing, 100080, People's Republic of China.

\section{Authors' information}

Qiong Liu, female, Qinzhou University College of Mathematics and Computer Science Professor. Master research circle tutor.

\section{Acknowledgements}

This work was supported by National Natural Science Foundation of China (10861003) and The Research Fund of Guangxi Higher Education of China (ZD2014137), Natural Science Foundation of Guangxi Province (No. 2016GXNSFAA380102).

Received: 6 May 2016 Accepted: 28 September 2016 Published online: 15 November 2016

\section{References}

1. Wang, ZL: The main plant diseases and insect pests and prevention and cure technology of balsam pear. Qinghai Pop. Agric. Sci. (4), 30 (2006)

2. Tang, SY, Xiao, YN: Single Population Biological Power System. Science Press, Beijing (2008)

3. Wang, MX: Chemical pesticide and environmental hormone. Rural Ecol. Environ. 15(4), 35-37 (1999)

4. Zhao, YF: The research progress of pesticide residues in food analysis. Foreign Med. 25(3), 173-177 (1998)

5. Wang, K: Random Biological Mathematical Model. Science Press, Beijing (2010)

6. Wei, CJ, Liu, Q: Pest management virus infection model. Syst. Sci. Math. 30(8), 1059-1069 (2010)

7. Fu, JB, Chen, LS: Pollution-free pest management strategy of mathematics research. Math. Practice Underst. 41(2), 144-150 (2011)

8. Jiao, JJ, Chen, LS: A pest management SI model with impulsive control concerned. J. Biomath. 22(3), 385-394 (2007)

9. Chen, HD: Impulse on the beneficial insects chemical control pest management model. J. Sun Yat-sen Univ. Natur. Sci. Ed. 49(3), 8-11 (2010)

10. Chen, YP, Liu, ZJ: Extinction and permanence of pest management SI model with twice impulses and Nonlinear incidence rate. J. Biomath. 24(4), 609-619 (2009)

11. DeBach, P, Rosen, D: Biological Control by Natural Enemies. Cambridge University Press, Cambridge (1991)

12. Grasman, J, van Herwaarden, OA, Hemerik, L, van Lenteren, JC: A two-component model of host-parasitoid interactions: determination of the size of inundative release of parasitoids in biological pest control. Math. Biosci. 169(2), 207-216 (2001)

13. Kidd, NAC, Jervis, MA: Population dynamics. In: Insect Natural Enemies. Springer, London (1996)

14. Chen, FD: Periodic solutions and almost periodic solutions for a delay multispecies logarithmic population model. Appl. Math. Comput. 171(2), 760-770 (2005)

15. Chen, LS: Pest control and geometric theory of semi-continuous dynamical system. J. Beihua Univ. Nat. Sci., 12(1), 1-9 (2011)

16. Liu, Q: The mathematical model of the red squirrel protection. Syst. Sci. Math. 33(9), 1083-1092 (2013)

17. Wang, L, Wang, MQ: Nonlinear Ordinary Differential Equation Qualitative Analysis. Harbin Industrial University Press, Harbin (1987) 